\title{
Viral vector delivers protective antibodies
}

\begin{abstract}
Influenza epidemics claim about 20,000 lives every year in the United States alone, particularly among the elderly and immunocompromised. The influenza virus evades the immune system by undergoing rapid antigenic evolution, and current vaccines have to be reformulated constantly. Now, reporting in Nature Biotechnology, David Baltimore and colleagues describe a new approach, termed vectored immunoprophylaxis (VIP), that is based on an adeno-associated virus (AAV) as a vector for broadly neutralizing antibodies. Importantly, and unlike vaccines, VIP does not require the
\end{abstract}

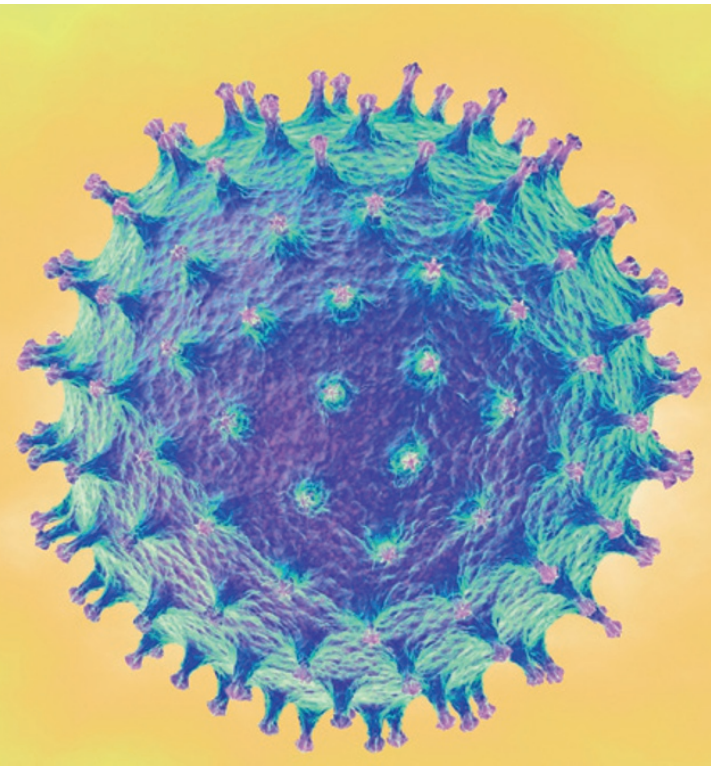

K-PHOTOS/Alamy initiation of an immune response, and therefore holds particular promise for the most vulnerable population groups.

The design of this approach was facilitated by recent studies that identified human antibodies with broadly neutralizing activity for diverse influenza strains. These were found to bind to conserved epitopes located on the stalk of the haemagglutinin (HA) protein. Vaccines based on these epitopes are being explored, but they still require the induction of an immune response and have only been moderately effective so far.

By contrast, the authors showed that a single intramuscular injection of an AAV-encoded broadly neutralizing antibody into BALB/c mice resulted in detectable levels of the human antibody within 1 week, and antibody production was maintained for the duration of the 64-week study. The neutralizing activity of sera from VIP-treated mice was tested in vitro, using five different green fluorescent protein (GFP)-tagged influenza reporter virions expressing HA proteins from three different HA subtypes. The sera from the VIP-treated mice neutralized multiple (often all) strains tested, and the level of neutralization correlated with the serum concentration of the AAV-encoded antibodies.

To test the protective effect of the antibodies, mice were challenged with three different $\mathrm{H} 1 \mathrm{~N} 1$ influenza strains (including the 2009 swine flu strain and a mouse-lethal H1N1 strain) 5 weeks after receiving VIP. As opposed to control mice, VIP-treated animals showed no weight loss or signs of illness. Protection was also tested in NOD. SCID-Il2 $\mathrm{rg}^{-/-}$(NSG) mice, which completely lack adaptive immune cells and have an impaired innate immune response. Young and old NSG mice expressed the AAVencoded antibody similarly well, and all VIP-treated mice were completely protected from influenza-induced weight loss and showed less inflammation and a lower viral load than control mice.

These results demonstrate that a single intramuscular injection of AAV is capable of protecting against diverse influenza strains, even in the immunocompromised setting. The authors argue that the broad efficacy could be particularly important during pandemics, when pre-existing immunity and conventional vaccines are unable to protect against newly emerging HA subtypes. Moreover, they point out that AAV is remarkably stable and can be lyophilized, so that VIP can be stockpiled before the start of a pandemic. And as new broadly neutralizing antibodies are discovered, the VIP approach can continuously be improved.

\section{Alexandra Flemming}

ORIGINAL RESEARCH PAPER Balazs, A. B. et al. Broad protection against influenza by vectored immunoprophylaxis in mice. Nature Biotech. 31 , 647-652 (2013) 